\title{
The Research of Ethical Consciousness for Physical Therapy Student
}

\author{
Myung-Chul Kim, PT, PhD • Dong-Hyeon Kim, $\mathrm{PT}^{\dagger} \cdot$ Chung-Joa Ahn, PT, MS \\ Department of Physical Therapy, Eulji University
}

Received: July 24, 2016 / Revised: July 30, 2016 / Accepted: August 10, 2016

(C) 2016 J Korean Soc Phys Med

\section{| Abstract |}

PURPOSE: The aim of this study was to examine the ethical values of physical therapy. This study was launched to establish a foundation for this field of study by gathering data about current topic of ethical values in the field of physical therapy.

METHODS: This investigation was conducted with 900 students from seven different universities in the Republic of Korea (three, four-year colleges. The methodology of this research was directed at revamping and supplementing ethical codes in the Korean Physical Therapy Association and establishing a set of ethics-related questions as inspired by the studies conducted by Youk and Choi. Each question was assigned a different point-value according to the standards of measurement that we established.

RESULTS: The scores of participants and their sibling status, it appears that whether participants had siblings did significantly impact their ethical outlook. The scores based on sibling one and two siblings, more than three siblings, no sibling were $29.42,29.03,28.25$, and 28.19 , respectively $(\mathrm{p}<.05)$. But in examining the average ethics scores with

†Corresponding Author : kdh88728@nate.com

This is an Open Access article distributed under the terms of the Creative Commons Attribution Non-Commercial License (http://creativecommons.org/licenses/by-nc/3.0) which permits unrestricted non-commercial use, distribution, and reproduction in any medium, provided the original work is properly cited. regards to reported past experiences with ethics education, we can see that past experience significantly impacted the results of the study on medical ethics perceptions. The scores corresponding to responses that one did or did not have prior experience were 29.40 versus 28.99 .

CONCLUSION: It is crucial to assure that physical therapy students receive well-founded information and proper ethical value. We suggest that create an ethics education program to enhance physical therapists and physical therapy students.

Key Words: Physical therapy, Ethics, Survey

\section{Introduction}

The definition of profession refers to those people who work in a specific field and who possess similar characteristics. They must have a systematic knowledge structure and exhibit professional organizational skills and be engaged in their profession, and they must receive approbation from those organizations that approve the profession, such that their individual values coincide with the respective field's basic social values. Moreover, there must be some general principles of ethics that direct the conduct of specialists in the respective professional field. Lastly, a professional culture under grids the conduct of 
the professionals (Ma, 2005; Kwon, 2003).

A physical therapist must perform one's duty with respect to the principles and the value of medical ethics. Even though one needs the requisite scientific knowledge and skills to perform physical therapy, a strong sense of ethics with regards to humanism and altruism is essential due in dealing with human beings.

The curriculum also in the educational process as well as physical aging can be psychological, to increase the level of knowledge of the social sphere should be strengthened (Ahn et al., 2014).

The guidelines for the World Confederation for Physical Therapy posit that ethics should be a major tenet of every single program (Maude et al., 2015). There should be college programs that teach medical ethics and that are aimed at changing the perspectives of physical therapists in the current medical system (Anne et al., 2014; Brockett, 1996). This sort of system would be able to cope with ethical issues that occur in the clinical field (Cho, 2008). However, in Korea, it is not easy for people to integrate medical ethics into their own physical therapy education. It means that when prospective physical therapy students become physical therapists and experience ethical dilemmas, they are often unable to perform their duties well, and they often struggle to provide the best and proper treatment for patients due to the difficulty they experience in making good decisions. It is possible to conclude that if physical therapy students were properly educated in medical ethics, they would earn have the capacity to handle any ethical problems in the future.

In this study, we attempt to identify the current place in which physical therapists and physical therapy students take action in order to determine what kind of ethical value both groups already possess in order to better understand the entirety of the situation, and so that we can ultimately establish a foundation of information for this field of study.

\section{Methods}

\section{Subject}

This study was conducted with 900 consenting students from seven different universities in South Korea (three and four grade). The collected data gathered from all participants was computed using the G-power 3.1.7 program.

\section{Research design}

This study was conducted using cross-sectional research and surveys to identify physical therapy students' medical ethics preconceptions and to understand current ethical consciousness. We received permission from seven different schools and used the post office delivery system to obtain the completed surveys.

We guaranteed that the survey takers' responses would be confidential according to the Statistics Act, and guaranteed that they would be protected under the privacy protection act.

1) The degree of consciousness of physical therapy ethics In this study, we used a tool that consists of ten questions based on the codes of ethics described by the Korean Physical Therapy Association to measure the degree of consciousness of physical therapy ethics. It was supplemented and revised according to the questionnaire created by Yook (1992), and Kim (2000). Survey takers chose one of the following responses: "Absolutely yes," "Somewhat yes," "Somewhat no," or "Absolutely no."

2) The degree of value of physical therapy ethics

In this study, we revised and utilized the tool that contains nine questions to measure the degree of value attributed to physical therapy ethics and that was made by Choi (2001), Choi (2010), and other researchers. Their tool is accompanied by a discussion of the current value of applied ethics and of implementing an ethics education 
into existing medical programs.

The gathered data through this study was calculated using a statistical analysis program called SPSS 21.0. We analyzed the data using the method described below.

First, the standard deviation, arithmetic mean, and frequent percentage were used to show the degree of general information of the subjects and the value of ethics on physical therapy.

Second, t-test was used to analyze the degree of the value of ethics on physical therapy students.

\section{Results}

\section{General information of the subject}

In this study, general information gathered on students who major in physical therapy is as follows (Table 1).

This study has found that $66.5 \%$ of participants were female students and $33.5 \%$ were male students. The total number of surveys we collected was 828. Most of the students were in their twenties $(63.6 \%)$, while $35.1 \%$ of the survey takers were teenagers and $.6 \%$ of respondents were in their thirties and forties; $45.4 \%$ of respondents came from metropolitan cities, while $31.8 \%$ came from small and medium-sized cities. Moreover, $15.1 \%$ were from Seoul and $7.7 \%$ came from rural areas, while $82.4 \%$ were currently taking courses at a four-year college and $17.6 \%$ were enrolled in three-year colleges. Of the respondents, $40.5 \%$ were freshman, $25.8 \%$ were sophomores, $18.8 \%$ were juniors, and $14.8 \%$ were seniors; $69.2 \%$ were middle class, $21.7 \%$ fell below the poverty threshold, and $9.1 \%$ were wealthy. Of the total, $64.1 \%$ of respondents did not claim to be religious, $18.6 \%$ were Christian, $11.0 \%$ were Buddhist, and $6.3 \%$ were Catholic. The percentage of respondents that had one sibling was $67.4 \%$, while $21.5 \%$ had two siblings; $6.3 \%$ were only children and $4.8 \%$ had more than three siblings; $75.2 \%$ of respondents had no family members who worked as a health professional.
Table 1. General information of the subject

\begin{tabular}{|c|c|c|c|}
\hline \multirow[b]{2}{*}{ Quality } & \multirow[b]{2}{*}{ Section } & \multicolumn{2}{|c|}{ PT students $(\mathrm{n}=828)$} \\
\hline & & $\begin{array}{c}\text { Real number } \\
(\%)\end{array}$ & $\mathrm{M} \pm \mathrm{SD}$ \\
\hline \multirow{2}{*}{ Gender } & Male & $277(33.5)$ & \multirow{2}{*}{$.472 \mathrm{a}$} \\
\hline & Female & $551(66.5)$ & \\
\hline \multirow{4}{*}{ Age } & $10 \sim 19$ & 291(35.1) & \multirow{4}{*}{.520} \\
\hline & $20 \sim 29$ & $527(63.6)$ & \\
\hline & $30 \sim 39$ & $5(.6)$ & \\
\hline & Over 40 & $5(.6)$ & \\
\hline \multirow{4}{*}{ Region } & Seoul & $125(15.1)$ & \multirow{4}{*}{.822} \\
\hline & Metropolitan city & $376(45.4)$ & \\
\hline & Small-medium city & $263(31.8)$ & \\
\hline & Rural area & $64(7.7)$ & \\
\hline \multirow{3}{*}{$\begin{array}{l}\text { Education } \\
\text { background }\end{array}$} & 3-year college & $146(17.6)$ & \multirow{3}{*}{.381} \\
\hline & 4-year college & $682(82.4)$ & \\
\hline & Master's degree & 0 & \\
\hline \multirow{3}{*}{$\begin{array}{l}\text { Economic } \\
\text { Status }\end{array}$} & Rich & $75(9.1)$ & \multirow{3}{*}{.541} \\
\hline & Not rich nor poor & $573(69.2)$ & \\
\hline & Poor & $180(21.7)$ & \\
\hline \multirow{4}{*}{ Religion } & Christian & $154(18.6)$ & \multirow{4}{*}{1.206} \\
\hline & Catholic & $52(6.3)$ & \\
\hline & Buddhism & $91(11.0)$ & \\
\hline & No religion & $531(64.1)$ & \\
\hline \multirow{4}{*}{ Siblings } & None & $52(6.3)$ & \multirow{4}{*}{.640} \\
\hline & One & $558(67.4)$ & \\
\hline & Two & $178(21.5)$ & \\
\hline & More than three & $40(4.8)$ & \\
\hline Medical & Yes & $205(24.8)$ & \multirow{2}{*}{.432} \\
\hline Professional & No & $623(75.2)$ & \\
\hline
\end{tabular}

${ }^{\mathrm{a}}$ Mean \pm Standard deviatio

However, $24.8 \%$ of respondents reported that they currently have at least one family member who is a health professional.

\section{The average scores on ethics according to the general information of the subjects}

In this study, the average scores of physical therapy students on ethics according to the general information of the subjects is as follows (Table 2).

The average ethics scores were ranked according to the general information of the physical therapy students. The total ethics score was 40 . In examining the average ethics 
Table 2. The average scores on ethics according to the general information of the subjects

\begin{tabular}{|c|c|c|c|}
\hline \multirow{2}{*}{ Quality } & \multirow{2}{*}{ Section } & \multicolumn{2}{|c|}{ PT students $(\mathrm{n}=828)$} \\
\hline & & $\mathrm{M} \pm \mathrm{SD}$ & $P$ \\
\hline \multirow{2}{*}{ Gender } & Male & $29.166 \pm 3.784 a$ & \multirow{2}{*}{.82} \\
\hline & Female & $29.225 \pm 3.361$ & \\
\hline \multirow{4}{*}{ Age } & $10 \sim 19$ & $28.969 \pm 3.227$ & \multirow{4}{*}{.37} \\
\hline & $20 \sim 29$ & $29.326 \pm 3.656$ & \\
\hline & $30 \sim 39$ & $30.800 \pm 2.864$ & \\
\hline & Over 40 & $28.600 \pm 3.286$ & \\
\hline \multirow{4}{*}{ Region } & Seoul & $28.952 \pm 3.724$ & \multirow{4}{*}{.79} \\
\hline & Metropolitan & $29.316 \pm 3.600$ & \\
\hline & $\begin{array}{l}\text { Small-medium } \\
\text { city }\end{array}$ & $29.171 \pm 3.284$ & \\
\hline & Rural area & $29.188 \pm 3.445$ & \\
\hline \multirow{3}{*}{$\begin{array}{l}\text { Economic } \\
\text { status }\end{array}$} & Rich & $28.773 \pm 4.305$ & \multirow{3}{*}{.29} \\
\hline & Not rich nor poor & $29.328 \pm 3.383$ & \\
\hline & Poor & $28.994 \pm 3.521$ & \\
\hline \multirow{4}{*}{ Siblings } & None & $28.192 \pm 3.248$ & \multirow{4}{*}{$.02 *$} \\
\hline & One & $29.423 \pm 3.437$ & \\
\hline & Two & $29.034 \pm 3.778$ & \\
\hline & More than three & $28.250 \pm 3.209$ & \\
\hline Medical & Yes & $29.224 \pm 3.651$ & \multirow{2}{*}{.93} \\
\hline Professional & No & $29.199 \pm 3.460$ & \\
\hline
\end{tabular}

${ }^{\mathrm{a}}$ Mean \pm Standard deviation $(\mathrm{p}<.05)$

score based on gender, it is clear that there is no correlation between gender and ethical values. The scores of both genders was 29.225 and 29.166. In examining the average ethics score based on the participants' region, it likewise appears as though region was not correlated with the ethical values. The scores based on location (metropolitan cities, rural areas, small and medium-sized cities, and Seoul) were $29.316,29.188,29.171$, and 28.952, respectively. The case was similar for economic status, in which the scores based on economic status (rich, neither-rich nor poor, and poor were $28.773,29.328$, and 28.994, respectively. Similarly, age did not have an impact on participants' ethical values. The scores based on age (forties, thirties, twenties, and teens) were $28.600,30.800,29.326$, and 28.969, respectively. However, when we examine the scores of participants and their sibling status, it appears that whether participants had siblings did significantly impact their
Table 3. Distribution of the average ethics score on the value of ethics

\begin{tabular}{|c|c|c|c|}
\hline \multirow{2}{*}{ Quality } & \multirow{2}{*}{ Section } & \multicolumn{2}{|c|}{ PT students $(\mathrm{n}=828)$} \\
\hline & & $\mathrm{M} \pm \mathrm{SD}$ & $P$ \\
\hline \multirow{3}{*}{ Values } & Absolutely clear & $30.351 \pm 3.794 a$ & \multirow{3}{*}{$.000^{*}$} \\
\hline & $\begin{array}{l}\text { Sometimes } \\
\text { confused }\end{array}$ & $29.065 \pm 3.260$ & \\
\hline & Case by case & $28.572 \pm 3.377$ & \\
\hline \multirow{3}{*}{$\begin{array}{l}\text { Ethics code } \\
\text { Knowledge }\end{array}$} & Know well & $30.358 \pm 3.740$ & \multirow{3}{*}{$.00^{*}$} \\
\hline & Know a little & $29.249 \pm 3.323$ & \\
\hline & Know little & $28.172 \pm 3.503$ & \\
\hline \multirow{2}{*}{$\begin{array}{l}\text { Education } \\
\text { Experience }\end{array}$} & Yes & $29.403 \pm 3.539$ & \multirow{2}{*}{.21} \\
\hline & No & $28.987 \pm 3.464$ & \\
\hline \multirow{3}{*}{$\begin{array}{l}\text { Current } \\
\text { Education } \\
\text { System }\end{array}$} & Enough & $29.865 \pm 3.579$ & \multirow{3}{*}{$.00^{*}$} \\
\hline & Not enough & $28.742 \pm 3.686$ & \\
\hline & Unsure & $29.068 \pm 3.247$ & \\
\hline \multirow{3}{*}{$\begin{array}{l}\text { Education } \\
\text { Opportunity }\end{array}$} & Yes & $30.445 \pm 3.321$ & \multirow{3}{*}{$.00^{*}$} \\
\hline & No & $26.531 \pm 3.505$ & \\
\hline & Unsure & $27.867 \pm 2.936$ & \\
\hline \multirow{3}{*}{$\begin{array}{l}\text { Ethical } \\
\text { Conflict }\end{array}$} & Yes & $29.660 \pm 3.526$ & \multirow{3}{*}{$.00 *$} \\
\hline & No & $27.817 \pm 3.508$ & \\
\hline & Unsure & $28.679 \pm 3.222$ & \\
\hline \multirow{2}{*}{$\begin{array}{l}\text { Education } \\
\text { Necessity }\end{array}$} & Yes & $29.352 \pm 3.500$ & \multirow{2}{*}{$.00^{*}$} \\
\hline & No & $27.727 \pm 3.223$ & \\
\hline
\end{tabular}

${ }^{\mathrm{a}}$ Mean \pm Standard deviation $(\mathrm{p}<.05)$

ethical outlook. The scores based on sibling (one sibling, two siblings, more than three siblings, no sibling were $29.423,29.034,28.250$, and 28.19 , respectively $(\mathrm{p}<.05)$. In examining the average ethics score with regards to whether or not respondents had a family member who worked in a medical health profession, it appears as if this fact did not significantly affect ethical values.

3. Distribution of the average ethics score on the value of ethics

In this study, the distribution of the average ethics scores of physical therapy students on the value of ethics is as follows (Table 3).

The ranking of the average ethics score is examined in this study according to the value of ethics of the physical therapy students. The total ethics score is 40 .

In examining the average ethics scores with regards to 
the level of ethics perception, we can see that ethical preconceptions significantly impacted the results of the study on medical ethics perceptions. The scores of the following responses, "clear perception," "confusing at times," and "depending on situation," were 30.351, 29.065, and 28.572 , respectively $(\mathrm{p}<.05)$. In examining the average ethics score regarding pre-existing knowledge of the code of ethics, we can see that this factor significantly impacted the results of the study on medical ethics perceptions. The scores corresponding to "knew a significant amount," "did not know much," and "knew some" were 30.358, 29.249, and 28.172 , respectively $(\mathrm{p}<.05)$. In examining the average ethics scores with regards to reported past experiences with ethics education, we can see that past experience significantly impacted the results of the study on medical ethics perceptions. The scores corresponding to responses that one did or did not have prior experience were 29.403 versus 28.987. In examining the average ethics scores with regards to the respondents' current educational situation, we can see that this factor significantly impacted the results of the study on medical ethics perceptions. The scores corresponding to responses "adequate," "unsure," “inadequate" were $29.865,29.068$, and 28.742 , respectively $(p<.05)$. In examining the average ethics scores with regards to the respondents' responses as to "they participated in education whether or not," we can see that this factor significantly impacted the results of the study on medical ethics perceptions. The scores corresponding to responses "yes," "not sure," or "no" were 30.445, 27.867, and 26.531, respectively $(\mathrm{p}<.05)$. In examining the average ethics scores with regards to respondents' reflections of ethical problems, we can see that this factor significantly impacted the results of the study on medical ethics perceptions. The scores corresponding to "yes," "not sure," and "no" were 29.660, 28.679 , and 27.817 , respectively $(p<.05)$. In examining the average ethics scores with regards to respondents' reflections on the necessity of ethics education, we can see that this factor significantly impacted the results of the study on medical ethics perceptions. The scores corresponding to "necessary" versus "unnecessary," and "no" were 29.352 versus 27.727 , respectively $(p<.05)$

\section{Discussion}

The purpose of this study was to provide a foundation of baseline data on medical ethics physical therapy students. The detailed goals are listed below. First, determine subjects' medical ethical conceptions upon assessing their general information. Second, determine the subjects' medical ethical values. Since there is no pre-study of the physical therapy ethics, so not enough data to compare the results obtained in this study. Therefore, consideration will be a brief description of the significance of the results of this study.

An analysis of the average ethics score according to the general information is shown below. After obtaining this information on physical therapy students, it was discovered that students' gender, region, economic status, age, or relationship to at least one medical professional was not significantly meaningful. However, the number of siblings was found the be. These results are probably the cause of the greater number of brothers will be more grown up mind that saving each other, this attitude is thought to have affected the ethical values.

The second questionnaire was ranking of the average ethics score is examined in this study according to the value of ethics of the physical therapy students. In this survey, except for the educational experience, the other all items were obtained a statistically significant. That means is ethics education experience was not affect the relationship between ethical values and ethics score. It is also a testament that much unaware of the importance of ethics, because they did not try to ethics training experience.

Overall, we it appears that most of the survey takers had strong ethical values, but that their ethics scores were 
not very high. Furthermore, those participants who received high scores tended to believe that it is necessary to participate in ethics education and that such education should be further developed in medical curriculums. Thus, we insist that it is crucial for physical therapy students to develop the appropriate knowledge of ethical values and for physical therapists to take special ethics courses to improve their personal ethical codes.

\section{Conclusion}

The aim of this study was to identify physical therapy students' medical ethics preconceptions and to understand current ethical consciousness. The research tool asked about the attitude toward physical therapy ethics and ten questions about the degree of knowledge and about the general information of the subjects. We have revised and used the tool that contained nine questions to measure the degree of value on physical therapy ethics. The scores of participants and their sibling status, it appears that whether participants had siblings did significantly impact their ethical outlook $(\mathrm{p}<.05)$. but In examining the average ethics scores with regards to reported past experiences with ethics education, we can see that past experience significantly impacted the results of the study on medical ethics perceptions. ( $<<.05)$. The scores corresponding to responses that one did or did not have prior experience were 29.403 versus 28.987 .

It is evident that overall, the entire group of subjects have high ethical values, but this is not necessarily reflected in their scores. We expect what for current and prospective physical therapists, the theory of ethics, the value of ethics, and the rule of ethics were integrated into physical therapy courses. If this were the case, physical therapy professionals would be better equipped to handle and solve ethical problems.

\section{References}

Ahn CJ, Kim MC, Kim SK, et al. tudy on Knowledge of the Elderly's Physical, Psychological and Social Aspects among College Students in Physical Therapy. J Korean Soc Phys Med. 2014;9(4):375-89.

Brockett M. Ethics, moral reasoning and professional virtue in occupational therapy education. Canadian Journal of Occupational Therapy. 1996;63(3):197-205.

Cho KW. evelopment of Ethics and Service Education Program for Students Majoring Health Care and Effect Analysis. The Korea Contents Society. 2008;8(10): 224-33.

Choi BL. Study on the biomedical ethics consciousness of the paramedic students. Master's Degree. Kongju university. 2010.

Choi YR. A Comparison of the Nursing Ethical Values between Clinical Nurses and Nursing Students. Master's Degree. Chonbuk university. 2001.

Hudon, A., Laliberté, M., Hunt, M., et al. What place for ethics? An overview of ethics teaching in occupational therapy and physiotherapy programs in Canada. Disability and rehabilitation, 2014;36(9):775-80.

Kim YS. Study on the comparison of consciousness on ethics between clinical nurses and student nurses. Master's Degree. Gyeongsang university. 2000.

Kwon SJ. A study on the consciousness of biomedical ethics of the nursing students and medical students. Master's Degree. Keimyung university. 2003.

Laliberté M, Hudon A, Mazer B, et al. An in-depth analysis of ethics teaching in Canadian physiotherapy and occupational therapy programs. Disability and rehabilitation. 2015;37(24):2305-11.

Ma MO. A study on the work ethics and behaviors of vocational rehabilitation workers. Master's Degree. Ewha womans university. 2005.

Yook JH. Nurse's ethics-level by nurses and patients. Master's Degree. Kyungpook university. 1992. 